\title{
A Importância Da Abordagem Sistêmica Na Ergonomia Para Um Design Funcional
}

Ourive-Assunçãos, Eliete Auxiliadora ${ }^{a}$; Figueiredo-Ourives-de, Attilio Bolivar ${ }^{\text {; }}$; Figueiredo-Gonçalves-de, Luiz Fernando ${ }^{c}$; Vieira-Horn, Milton Luiz ${ }^{d}$; Moreira, Isabel Cristina Victoria $^{\mathrm{e}}$ \& Castro-Gómez, Francisco ${ }^{\mathrm{f}}$

${ }^{\text {a }}$ Pós-doutoranda - Estudante ( Núcleo de Abordagem Sistêmica do Design, Universidade Federal de Santa Catarina, Brasil ), elieteourives@gmail.com,

${ }^{\mathrm{b}}$ Graduando - Estudante (Núcleo de Abordagem Sistêmica do Design, Universidade Federal de Santa Catarina, Brasil), gastronomia.cosine@gmail.com,

${ }^{\mathrm{c}}$ Pós-doutor - Professor (Núcleo de Abordagem Sistêmica do Design, Universidade Federal de Santa Catarina, Brasil), lffigueiredo2009@gmail.com,

d Doutor - Professor (Laboratorio Lab Design, Universidade Federal de Santa Catarina, Brasil), milton@cce.ufsc.br,

${ }^{\mathrm{e}}$ Graduando - Estudante (Núcleo de Abordagem Sistêmica do Design, Universidade Federal de Santa Catarina, Brasil), gastronomia.cosine@gmail.com,

${ }^{\mathrm{f}}$ Doutor - Professor (Núcleo de Abordagem Sistêmica do Design, Universidade Federal de Santa Catarina, Brasil) and francisco.gomez.castro1@gmail.com.

\begin{abstract}
The systemic approach is an interdisciplinary process, whose main principle is to understand the interdependence and relationships of all areas and the need for their integration, allowing a closer approximation between their limits of study. In this context, the systemic view of ergonomics, especially with regard to safety, comfort and effectiveness of use, functionality and operability of objects, considering all products or systems of products, as a system of use, from the very simple to the most complex or systemic, aims to adapt them to human beings, in view of the activities and tasks performed by them. Regarding functional design, the ergonomics knowledge, in this systemic view, related to its design methodology, is absolutely necessary, and its application points out the better suitability of the products to its users. As is the case with functional women's clothing, especially with regard to breast protection, which are conventional parts that require a correct sizing and
\end{abstract}


specification of fabrics and other materials. It is a type of clothing that has diverse functionality, such as for physical protection, breast enlargement, cloth filling, Y790 water, oil, structured with wire, etc .; for breastfeeding (a bra that opens in front, partially or totally); for the inclusive design (people with disabilities and reduced mobility, in the case of breasts with prosthesis or bracing) facilitating with closings and openings placed on pieces of clothes difficult to handle, comfortable clothes and easy to wear. They are pieces worn by people with variable anthropometric biotypes and percentiles and with body characteristics that change significantly in the passages for adolescence, adulthood and the elderly. The body changes present significant differences in terms of breast volume, in which the ergonomic solutions through a systemic approach that is more evident to the complexity of use are the most necessary in terms of attributes such as safety, comfort, body comfort, ease dress, functionality, and aesthetics. This research, although exploratory and descriptive, is not free of challenges, has the objective, through data and ergonomic information systemic contribute to the functional design, so as to offer subsidies for the production of functional clothing or wearable technology, with the attributes cited, respecting the diversity and inclusion of people in all phases of their life, thus meeting the formal principles of design.

Key words: Systemic approach, Ergonomics, Functional design

\section{Resumo}

A abordagem sistêmica é um processo interdisciplinar, cujo princípio primordial é compreender a interdependência recíproca e relações de todas as áreas e da necessidade de sua integração, permitindo maior aproximação entre os seus limites de estudo. Nesse contexto o olhar sistêmico, da ergonomia, sobretudo no que se refere à segurança, ao conforto e à eficácia de uso, de funcionalidade e de operacionalidade dos objetos, considerando todos os produtos ou sistemas de produtos, como sistema de uso, desde os mais simples aos mais complexos ou sistêmicos, tem como objetivo adequálos aos seres humanos, tendo em vista as atividades e tarefas exercidas por eles. No que se refere ao design funcional, os conhecimentos da ergonomia, nessa visão sistêmica, relativos à sua metodologia de projeto, são absolutamente necessários, e a sua aplicação aponta a melhor adequação dos produtos aos seus usuários. Como é o caso do vestuário feminino funcional, sobretudo no que se refere a proteção das mamas, que são peças 
convencionais que necessitam de um correto dimensionamento e especificação dos tecidos e de outros materiais. É um tipo de vestuário que apresenta funcionalidade diversa, como para a proteção física, o aumento do volume da mama, enchimento no bojo de pano, deY790 água, de óleo, estruturado com arame, etc.; para amamentação (sutiã que se abre na frente, em parte ou totalmente); para o design inclusivo (pessoas com deficiência e mobilidade reduzida, no caso de mamas com prótese ou órtese) facilitando com fechamentos e aberturas colocadas em peças de roupas dificeis de manusear, roupas confortáveis e fáceis de vestir. São peças usadas por pessoas com biótipos e percentis antropométricos variáveis e com características corporais que mudam significativamente nas passagens para a adolescência, idade adulta e idosa. As mudanças corporais apresentam diferenças significativas em termos de volume das mamas, nas quais as soluções ergonômicas por uma abordagem sistêmicas que se evidencia mais para a complexidade de uso, são as mais necessárias em termos de atributos como, segurança, conforto, comodidade corporal, facilidade do vestir, funcionalidade, além da estética. Esta pesquisa, embora exploratória e descritiva, não isenta de desafios, tem por objetivo, por meio de dados e informações ergonômicas sistêmicas contribuir com o design funcional, de modo a oferecer subsidios para a confecção de roupas funcionais ou tecnologia vestivel, com os atributos citados, respeitando a diversidade e inclusão das pessoas em todas as fases de sua vida, atendendo assim os princípios formais do design.

Palavra-chave: Abordagem sistêmica, Ergonomia, Design funcional.

\section{Introdução}

Com a presença das mulheres no ambiente da produção, a literatura tem relatado as mudanças ocorridas no design do vestu $\mathrm{r}$ io minino $\mathrm{s} j \mathrm{pl}$ simpli $\mathrm{i}$ o o vstir ou pl ms uliniz o o tr j us on orto $t$ orr nts tors so $i$ is econômicos e produtivos. Verifica-se que as discussões têm se concentrado com maior ênfase nos aspectos estéticos da configuração da roupa, inerentes aos desdobramentos da moda, na finalidade de buscar sua real funcionalidade ajustada a antropometria do corpo feminino. 
Neste contexto, com diversos modelos, as roupas de compressão, por exemplo, estão sendo utilizadas em diversas modalidades esportivas. Elas pretendem ajudar a manter a postura correta, manter a temperatura corporal ideal e diminuir o atrito entre os músculos. Os modelos variam muito, para os países frios eles mantém o calor, para os quentes, deixam o suor evaporar e possuem tecidos que deixam a pele respirar, além das costuras que são baixas para não machucar a pele. As roupas viraram aliadas na qualidade de vida e do bemestar, com a proliferação do chamado vestuário inteligente, visando a atividade de projeto para roupas funcionais.

A utilização de tecnologia para potencializar o vestuário feminino, tornando-o eficiente, interativo e funcional, encontra-se em evolução. Nesse aspecto, a pesquisa e estruturação de informações são importantes para estabelecer diretrizes de requisitos de projeto, formando uma base de dados referente às roupas funcionais. Essa base de dados irá contribuir para melhorar as roupas funcionais e com isso minimizar o desconforto do movimento das mamas das mulheres durante a realização de atividades físicas - desenvolvidas em chão de fábrica e práticas desportivas, como andar rapidamente, correr e alguma outra atividade que envolva o movimento das mamas. Além disso, esse desconforto geralmente, segundo as mulheres, aumenta durante o período menstrual.

Neste sentido, a relevância deste estudo pode ser estabelecida em consequência de dois parâmetros específicos: o primeiro voltado para a área acadêmica específica na qual a discussão teórica é desenvolvida; e, o segundo, a pesquisa, o qual explora o uso de mapas mentais na estruturação e planejamento de informações, apresentando diretrizes do processo de análise direcionado a construção lógica de ações na pesquisa de roupas funcionais feminina.

Através do primeiro parâmetro, buscam-se instrumentos com a finalidade de realizar um controle dos procedimentos efetuados no processo, identificando as oportunidades de aperfeiçoamento na pesquisa de roupas funcionais feminina. $\mathrm{O}$ segundo parâmetro, o estudo prático, fortifica-se e integra-se mais no ambiente de pesquisa científica, pois através da aplicação prática, utilizando os mapas mentais, apresenta e demonstra a importância da necessidade de sistematização do tema, contribuindo no processo de busca de informações importantes para o direcionamento de ações sobre a pesquisa de roupas funcionais femininas.

O presente estudo, ao enveredar neste campo de pesquisa, estará contribuindo para buscar diretrizes que possam orientar o processo de pesquisa, assim como, permitirá gerar discussões práticas sobre o tema, ajudando o seu desenvolvimento e aproximando a ciência da realidade acadêmica. 


\section{Procedimentos metodologicos}

De acordo com Gil (2010), quanto à natureza da pesquisa, o presente trabalho utiliza uma metodologia de pesquisa aplicada, pois busca gerar conhecimento para aplicações práticas de soluções a problemas específicos; quanto à abordagem, como pesquisa qualitativa, pois visa qualificar os dados coletados, conforme afirma Richardson et al (1999). E, quanto aos objetivos, como pesquisa exploratória, pois tem como finalidade desenvolver, esclarecer e modificar conceitos e ideias, proporcionando maior familiaridade com o problema (Gil, 2010).

\section{Abordagem sistemica}

Para este novo olhar do design pôde-se entender o processo como sendo uma abordagem sistêmica, ou seja, o estudo global dos sistemas de forma a envolver todas as suas interdependências, pois cada um dos elementos, ao serem reunidos para construir uma unidade funcional maior, desenvolvem qualidades que não se encontram em seus componentes isolados (Bertalanffy, 2008).

Sendo assim, o designer atua com uma nova postura, com foco ampliado na atividade do projeto para a cultura projetual, da tecnologia para a cultura tecnológica e da produção para a cultura produtiva, evoluindo em direção a uma perspectiva sistêmica (Krucken, 2009).

Neste tipo de abordagem os objetos dão lugar aos sistemas. Em vez de essências e de substâncias, a organização; em vez das unidades simples e elementares, as unidades complexas; em vez dos agregados formando corpos, os sistemas de sistemas de sistemas (Morin, 2005). A abordagem sistêmica que está presente no processo do design mostra como deve acontecer o trabalho dentro do processo de criação de um objeto, para que o mesmo seja aperfeiçoado, entendendo que cada parte possui sua importância e deve estar presente dentro de um sistema maior.

A abordagem do design busca a produção de coerência, e tem como critério de sucesso a satisfação da sociedade (Bonsiepe, 2011). Dessa forma seus resultados podem se caracterizar como uma "inovação sociocultural" (...) é a percepção sistêmica que caracteriza e estimula a atuação do design na contemporaneidade (Krucken, 2009).

Dessa maneira, entende-se que as transformações desencadeadas pelo aperfeiçoamento das novas tecnologias visam compreender como cada parte de um estudo pode se interrelacionar separadamente dentro de um sistema holístico, a fim de mudar as formas de pensar nos projetos. 
"O proj to port $\mathrm{n}$ to $\mathrm{t} \mathrm{m}$ qu $\mathrm{s} r$ r to novos $\mathrm{v} n$ tos $\operatorname{prv} \mathrm{n} \quad \mathrm{o} \quad \mathrm{n} \quad$ ss $\mathrm{i}$ de de mudanças e incorporando o usuário como participante ativo da solução que será proposta. A variedade de serviços que foram desenvolvidos a partir da evolução da tecnologia da in orm o ( ) r or $n$ ssi $1 \times$ i ili no proj to" $(K r u k n, 2009)$

Levando em consideração os sistemas existentes, nota-se que o design está inserido nos sistemas de informação, que de acordo com Lyytinen (1996, p. 14 apud Gómez, 2004) é considerado como uma

Institui o o muni tiv qu $\mathrm{s}$ ss $\mathrm{mlh}$ "um $\mathrm{m}$ io comunicação de massa para um grupo lo l" $\mathrm{Pr}$ o utor $\mathrm{ri} \quad \mathrm{o} \quad$ sist $\mathrm{m}$ in orm o $\mathrm{ri}$ orig $\mathrm{m}$ ou atualização a uma instituição de comunicação que intervém no meio social por meio de tivi $\mathrm{s} \quad \mathrm{mo} 1 \mathrm{~g} \mathrm{~m}$ in orm ion $\mathrm{l}$ : "um sist $\mathrm{m}$ in ormação é um instrumento linguísti o o muni o"

Isso demonstra que o design é um instrumento linguístico de comunicação que intervém no meio social com seus projetos focados na melhoria da qualidade de vida da sociedade e do mundo. É por este motivo, que o design busca constantemente comunicar uma informação por meio de um produto/serviço. Assim informa Santos (2000) que propõe uma abordagem na qual o design é considerado como um sistema processador de informações.

Com isso, é perceptível que o resultado do processo de design não é o produto ou projeto, e $\operatorname{sim} \mathrm{m}$ is in orm õ "qu iro $\mathrm{i} \mathrm{nti} \mathrm{i} \quad \mathrm{tr} \min$ o pro uto ou $\mathrm{mpr} \mathrm{s}$ no $\mathrm{mr}$ o posicionando-o o mrl o onorrnts o nsumi or s" ( $\mathrm{S} n$ tos 2000) Além isso s percebe como a abordagem sistêmica se faz presente no design, pois além da entrada, processo e saída da informação, ainda possui o feedback da interação do usuário com o produto/serviço, formando assim um sistema aberto. Assim confirma Vasconcellos (2013) informando que a noção de $\quad \mathrm{k}$ (ou r tro $\quad$ o $\quad$ ou $r$ tro lim $\mathrm{t} \quad$ o $\quad$ o sist $\mathrm{m}$ ) "qu r dizer que uma parte do efeito (saída ou output) ou do resultado do comportamento/funcionamento do sistema volta à entrada do sistema como informação (entrada ou input) e vai influir sobre o seu o mport m nto su s qu nt"

\section{A ergonomia e o vestuário}

Iida (2005) define a ergonomia como a ciência que estuda a adaptação do trabalho ao homem, sendo que o significado de trabalho envolve o estudo da relação entre o homem e fatores como, os equipamentos, o ambiente de trabalho, as máquinas, as instalações e as atividades desenvolvidas e a aplicação dos conhecimentos de anatomia, físiologia e psicologia, na solução dos problemas surgidos desse relacionamento. Para o autor, nesse conceito, a ergonomia é abordada como uma ciência multidisciplinar, por fazer uso de diversas áreas do conhecimento, no campo das ciências humanas, biológicas, tecnológicas, 
relacionando-se com os profissionais que desenvolvem produtos a serem utilizados pelo homem.

O domínio esp i lis t rgonomi é $\mathrm{m} \quad \mathrm{rng} \mathrm{nt}$ pois "tr $\mathrm{t}$-se de uma disciplina ori $\mathrm{t} \quad \mathrm{pr}$ um or $\mathrm{g} \mathrm{m}$ sistêmi to os os $\mathrm{sp}$ tos tivi hum $\mathrm{n}$ " (Abergo, 2017). Diante da abrangência dessa dimensão verifica-se a necessidade de uma abordagem holística de toda área de ação da disciplina e relação aos aspectos físicos, cognitivos, sociais, organizacionais, ambientais, etc. (Abergo, 2017).

Deste modo, o objeto da Ergonomia, seja qual for a sua linha de atuação, ou as estratégias e os métodos que utiliza, é o homem no seu trabalho, realizando a sua tarefa cotidiana, executando as suas atividades. Pois, os objetivos práticos da ergonomia são a segurança, a satisfação e o bem-estar dos trabalhadores e clientes no seu relacionamento com sistemas produtivos e com os produtos propriamente ditos.

Em se tratando da relação da Ergonomia e produtos de vestuários, Reilly (2010), demonstram que no projeto devem ser enfatizados fatores como, isolamento para proteger contra o frio e calor; permeabilidade ao vapor e ao ar em que se verifica a capacidade de perda de calor; resistência ao vapor; e a proteção contra: incêndio, penetração de água, poluentes e produtos químicos líquidos por meio da impermeabilização. Considerando também, a visibilidade das peças de vestuário e suas propriedades mecânicas.

Portanto, ao se projetar um produto para o vestuário, deve-se agregar a ele algumas funções e conceitos básicos, capazes de atender necessidades, transmitir segurança, bem como, proporcionar praticidade e conforto ao usuário. E, para que isso ocorra, os princípios de fabricação devem estar centrados no usuário e permeados por estudos ergonômicos. Pois, o vestuário, mantém com o usuário uma efetiva relação de uso no que diz respeito à proteção, ornamentação ou pudor. Ele faz parte do meio físico e material, antes da pessoa interagir com qualquer objeto, ou seja, é uma extensão do próprio corpo, interferindo na realização das ações do usuário de forma positiva ou negativa (Montemezzo, 2003).

Entretanto, observa-se algumas restrições em relação a esses parâmetros ergonômicos nos projetos de produto de vestuário desenvolvidos industrialmente, que utilizam tabelas de medidas com o perfil do usuário. Algumas restrições de destaque para este trabalho estão: a intima relação estabelecida entre o produto e o corpo humano, a diversidade de estilos e peças lançadas no mercado sem testes de aceitação do usuário. Um dos exemplos é o das roupas íntimas, nas quais as soluções ergonômicas inteligentes são as mais necessárias em termos de segurança, conforto e comodidade corporal, além da facilidade do vestir, correto dimensionamento e especificação dos tecidos e de outros materiais e ao próprio design dos diversos modelos, peças e aviamentos que configuram esses tipos de vestimenta. Pois, são peças usadas por pessoas com biótipos e percentis antropométricos (critério utilizado para 
classificar as dimensões do corpo humano para diferentes tamanhos) extremamente variáveis e com características corporais que mudam significativamente nas passagens para a adolescência, idade adulta e provecta, com diferenças significativas em termos de volume corporal, no caso das mamas femininas.

Esses dados ergonômicos, por sua vez, vão influenciar o design dessas roupas, consubstanciados em grande variedade de tipos de produtos como, por exemplo, os sutiãs em seus formatos taça, 1/2 taça, triangular e outros. Os sutiãs apresentam funções diversas: de simples proteção física; para aumento do volume do seio - enchimento no bojo de pano, de água, de óleo; estruturado com arame etc.; para amamentação (sutiã que se abre na frente, em parte ou totalmente); e até os especiais. Complementando o sutiã, as alças (retas, cruzadas, transparentes, removíveis e outras) apresentam também variadas características, como, além de seu próprio formato, vários tipos de dispositivos de fixação, ajustes e regulagens, que permitem o uso de diferenciados decotes nas roupas.

O design dessas peças deve apresentar soluções de configuração segura, confortável e, sobretudo, funcional, independentemente do estilo estético formal. Elas precisam atender às demandas dos usuários, referentes a essas características, pois, conforme Labat (2006), o designer de vestuário deve entender o funcionamento no que se refere à interface entre o corpo e o vestuário e a relação entre vestuário e corpo existe a necessidade direta de se obter conforto. A demanda de conforto, muitas vezes, não é atendida em detrimento do desejo de estar acompanhando as tendências sazonais de moda. Isso gera agressões ao corpo, podendo ocasionar disfunções ou deformações físicas de diferentes graus. Os estudos de Labat (2006) demonstram essas constatações, quando ao verificar que as maiorias dos estilistas priorizam os elementos visuais dos produtos e o funcionamento físico do corpo não é considerado com ênfase.

Labat (2006) defende a ideia que projetar compreende uma perspectiva em que a ergonomia fornece subsídio para o designer atender às necessidades física, psicológica e social do usuário. Isso permite selecionar componentes adequados para cada forma, materiais e detalhes utilizados no vestuário. Por meio da combinação desses componentes que atendam fatores ergonômicos, o designer pode aumentar o conforto, segurança e desempenho do produto. Para alcançar um nível de conforto compatível entre o corpo humano e o vestuário, os princípios de fabricação devem estar centrados no usuário.

Assim, os designers de vestuário que incorporam a ergonomia em seu processo de projetação e consideram todos os elementos físicos que afetam o conforto, a visibilidade, as limitações auditivas e o equilíbrio térmico. $\mathrm{O}$ uso da ergonomia permite que se crie a roupa mais apropriada melhorando as propriedades aerodinâmicas ao desempenhar suas atividades.

\section{O design funcional}


Design, estética ou funcionalidade, a eterna rivalidade em cada peça que se compõe. O que é o design? O design tem que ser funcional? Ou a estética assume maior peso? O que importa num processo de concepção e idealização de uma peça?

Uma das melhores definições do que é Design Funcional é aliar estética e praticidade. Por exemplo um eletrodoméstico, para ter um design funcional, tem que ser esteticamente agradável e de uso fácil e prático. E isso pode ser aplicado a qualquer objeto ou ambiente. Um espaço para ter o mesmo conceito tem que garantir a facilidade do uso, tornando a interação uma tarefa simples, objetiva e agradável, e, muitas vezes, isso está atrelado ao aproveitamento prático e inteligente de pequenos espaços. Uma roupa para ser funcional tem que aliar conforto e beleza. Para o design funcional é muito importante que a experiência do usuário não seja subestimada.

Pois, design nada mais é que a melhoria dos aspectos funcionais, ergonômicos e visuais do produto, de modo a atender às necessidades do consumidor, melhorando o conforto, a segurança e a satisfação dos usuários. Porém, cada designer ou pessoa comum tem a sua visão do que deve ser o design. Uns entendem que o design tem que se destacar pela sua beleza e pelo seu aspecto, enquanto outros esquecem por completo a estética e voltam-se p r o imp to qu su orm po ss umir Exist ntr outros o " sign u tor" uj peça é caracterizada pelo seu criador, o design que é arte, ou seja, que se transforma numa peça de arte e o design pela funcionalidade.

O design é caracterizado como um campo híbrido que atua na conexão entre informação, artefato, usuário e sistema, tendo como natureza a atividade de projeto, capaz de viabilizar soluções sistêmicas e criativas para os desafios que são propostos.

Entendemos o design com uma diretriz Função-Forma-Estética. Simplificando a ideia, temos como pilar, ao conceber cada uma das nossas peças, a perspectiva de design funcional. Não criamos peças para não serem utilizadas, pelo contrário. Para nós, o design faz sentido e existe para melhorar o nosso quotidiano, através do que se cria, com imaginação e criatividade.

De acordo com Bonfim (1995), a atividade de projeto envolve três aspectos principais, os objetivos que vão determinar "para que" ou "para quem" é o projeto, o conteúdo que prsnt xpli o o "o qu" o proj to tr vés intrprt o os o j tivos o estabelecimento das funções e da estrutura do produto e a metodologia de projeto que mostr "o mo" o proj to s r s n volvi o A igur 1 prsnt sssinorm õ 


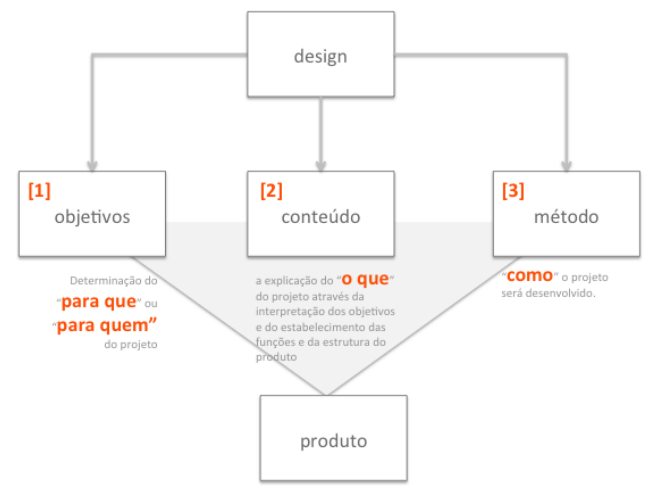

Fig. 1 - A atividade de projeto envolve três aspectos principais.

A metodologia de projeto enfatiza várias etapas, conforme parte da identificação de um problema, após vários tipos de análise é que se define o problema e se realiza o anteprojeto, geração de alternativas e chegando ao produto. A figura 2 apresenta uma síntese de vários conceitos do processo de design.

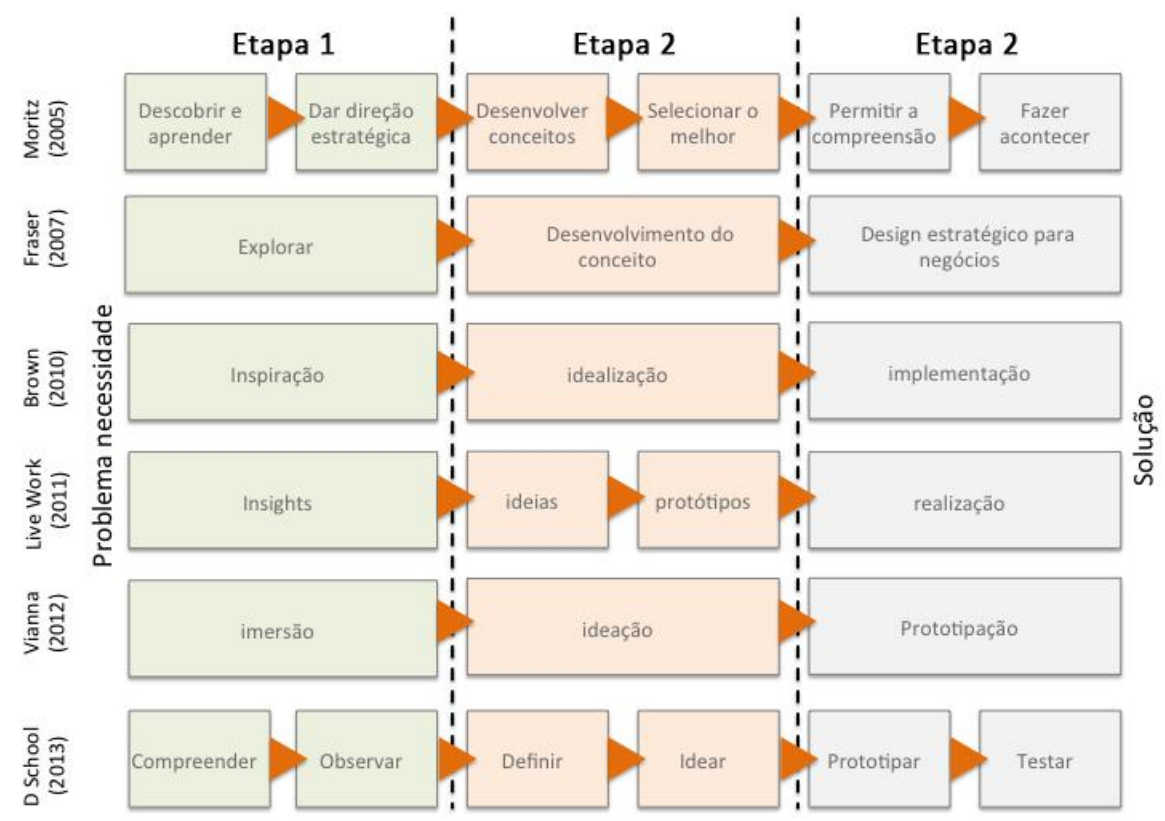

Fig. 2 - Síntese de vários conceitos do processo de design. 
Ourives, Eliete Auxiliadora Assunção; Figueiredo, Attilio Bolivar Ourives de; Figueiredo, Luiz Fernando Gonçalves de; Vieira, Milton Luiz Horn; Moreira, Isabel Cristina Victoria \& Castro,

\subsection{A roupa funcional e seu contexto histórico}

O debate a respeito dos aspectos funcionais do vestuário tem alimentado a indústria da moda na disputa conceitual entre forma e função. A forma até agora tinha levado vantagem no que se refere ao design de produtos de moda. Porém, nos dias atuais estimula-se o reconhecimento do ganho que o usuário pode ter ao vestir uma roupa funcional, evidenciando a importância da função da roupa.

A roupa funcional são roupas fabricadas com uma modelagem e tecidos cientificamente desenvolvidos para trazer benefícios úteis para a saúde e para a qualidade de vida das pessoas, indo muito além das antigas funções estéticas.

A preocupação com a funcionalidade da roupa feminina pertence a um passado longínquo, aproximadamente 400 a.c. e passou por várias fases (vide figura 3).
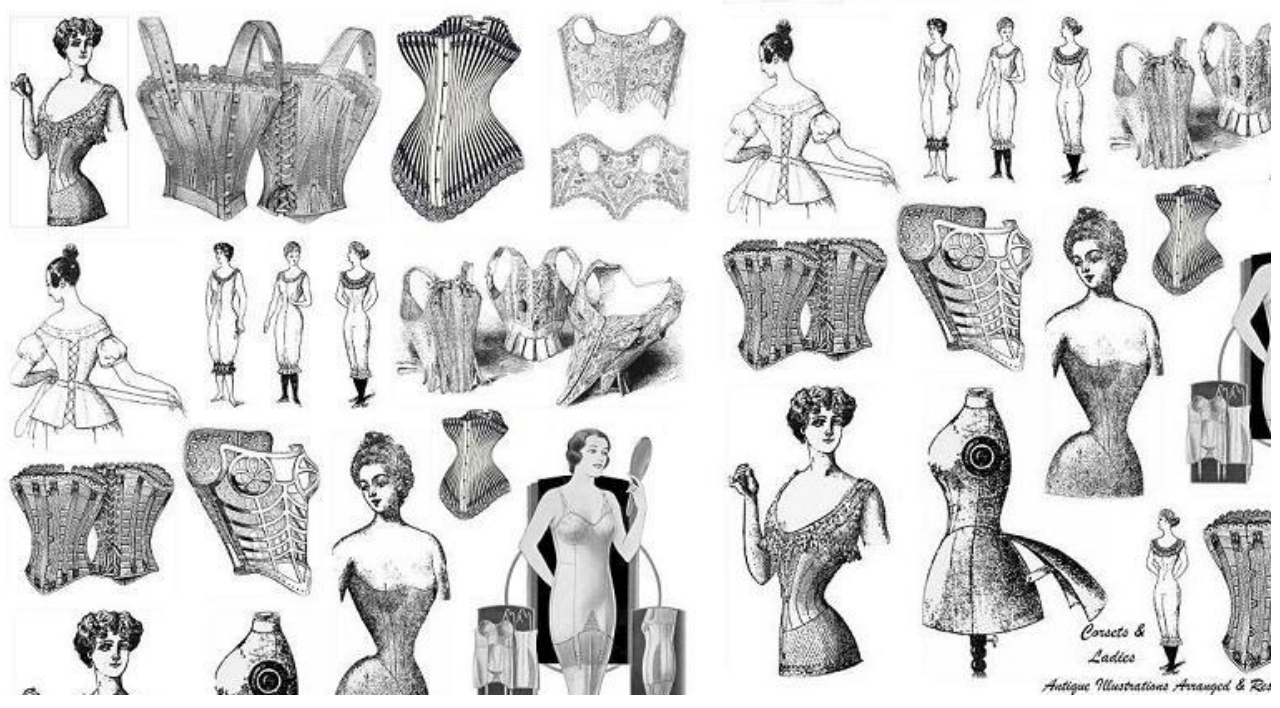

Fig 3 - Fases (maishistoria, 2017)

O primeiro registro de roupa específica para os seios femininos foi uma contribuição dos gregos, através de uma faixa de linho que envolvia o busto, chamada de mastodeton (vide figura 4), e que tinha a função de cobrir e segurar os seios. (Ewing, 1989). 


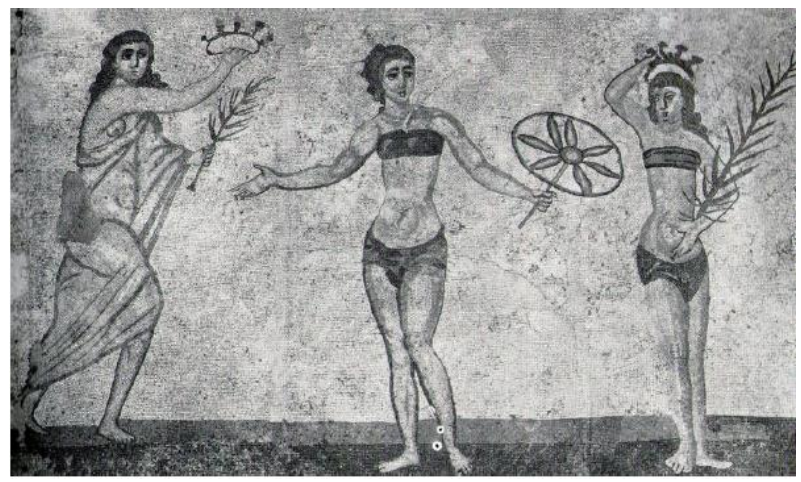

Fig 4 - Mosaico de Villa Armena, Sicília, 400 a.C. (Ewing, 1989)

Entre 1750 a.C e 1400 a.C., surgiu algo parecido com o espartilho que apertava a região do abdômen e ia até a altura dos seios. $\mathrm{O}$ uso deste tipo de roupa íntima variava em modelos (vide figura 5) foi até o século XX. (Ewing, 1989).

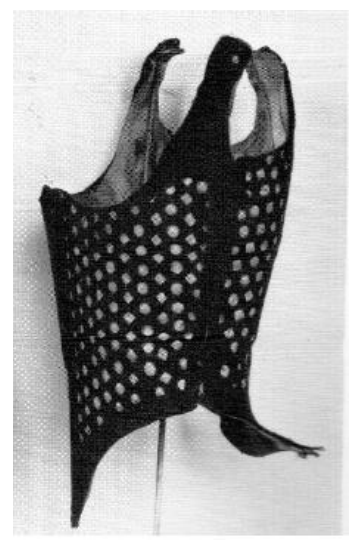

Fig 5 - Corset do século XVI (Ewing, 1989).

Em 1889, Herminie Cadoll decidiu cortar em duas partes o tradicional espartilho, dando as primeiras configurações do que viria a ser o primeiro sutiã (vide figura 6). 


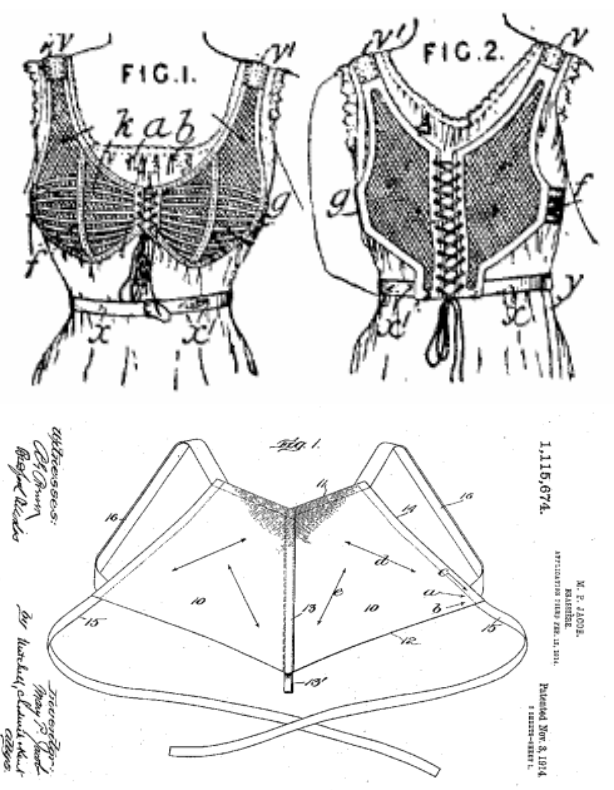

Fig 6 - Primeira configuração e BaklessBra. (Maishistoria, 2017)

No século XX, por volta de 1914, surge a preocupação com o conforto dos seios femininos, tr vés o protótipo $\mathrm{mri} n \mathrm{M}$ ry $\mathrm{Ph} \operatorname{lps} \mathrm{J}$ o nomin on épo " $\mathrm{rssir}$ " Ela criou a partir de lenços um protótipo que era macio, curto e acomodam separadamente os dois seios, esse modelo era chamado de BaklessBra como mostra a figura 6 (EWING, 1989). A forma Brassiere percorreu décadas e transformações (vide figura 7).

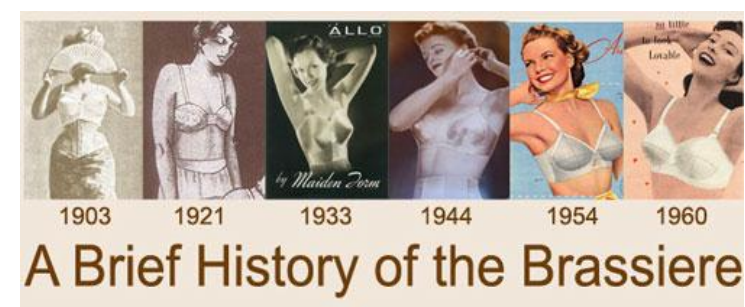

Fig 7 - Brassiere. (Maishistoria, 2017) 
Em 1926, surge o modelo criado por Rosalin Klin, constituído por duas copas transpassadas na frente, com caimento e uso de elásticos. Porém foi o modelo de Kestos, o primeiro a modelar os seios através de copas (vide figura 8) (EWING, 1989).

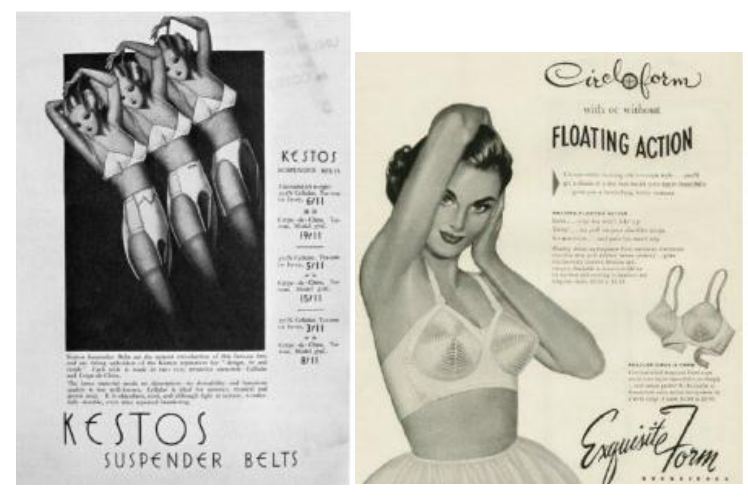

Fig 8-BrassiereKestos e sutiã cone. (Ewing, 1989).

A partir de 1935 e utilizado até os dias atuais, a Warner Bross Corset Company introduziu no mercado uma numeração diferenciada para sutiãs, com base nas medidas do tórax e do busto separadamente. As copas tinham quatro tamanhos diferentes A, B, C e D. Na década de 50, houve a predominância dos sutiãs com formato de cone (vide figura 8).

Já na década de 70 ocorre o lançamento do primeiro sutiã de bojo, conhecido como "won $\mathrm{r} r$ " E surg $\mathrm{m} 1977 \operatorname{mos} \mathrm{mr}$ Vitori's $\mathrm{S} r \mathrm{t}$ sp í i $\mathrm{n}$ mo íntima. E, a partir de 1990 e até os dias atuais, existe no mercado várias opções, para várias finalidades e funções.

\subsection{Sistematização da roupa funcional usando o mapa mental}

O méto o o nh i o o mo "M p M nt l" oi ri o p lo inglês Tony Buz n é 70 , e é adotado por profissionais das mais variadas áreas do conhecimento. Ele tenta representar, de forma gráfica, como as ideias se organizam e se associam em torno de um elemento principal, criando uma linha de raciocínio muito mais fluida, lógica e espontânea. Os Mapas Mentais são perfeitos para o planejamento, cada ponto no mapa pode aprofundar numa área em particular relacionada com o tema principal. (BUZAN, 2005; 2009). A figura 9 apresenta a estrutura de um mapa mental e a figura 10 um exemplo de como criar um mapa mental. 
Ourives, Eliete Auxiliadora Assunção; Figueiredo, Attilio Bolivar Ourives de; Figueiredo, Luiz Fernando Gonçalves de; Vieira, Milton Luiz Horn; Moreira, Isabel Cristina Victoria \& Castro,

Francisco Gómez

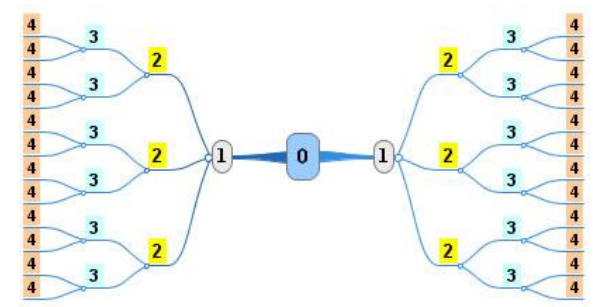

Fig 9-A estrutura de um mapa mental. (mapasmentais, 2017)

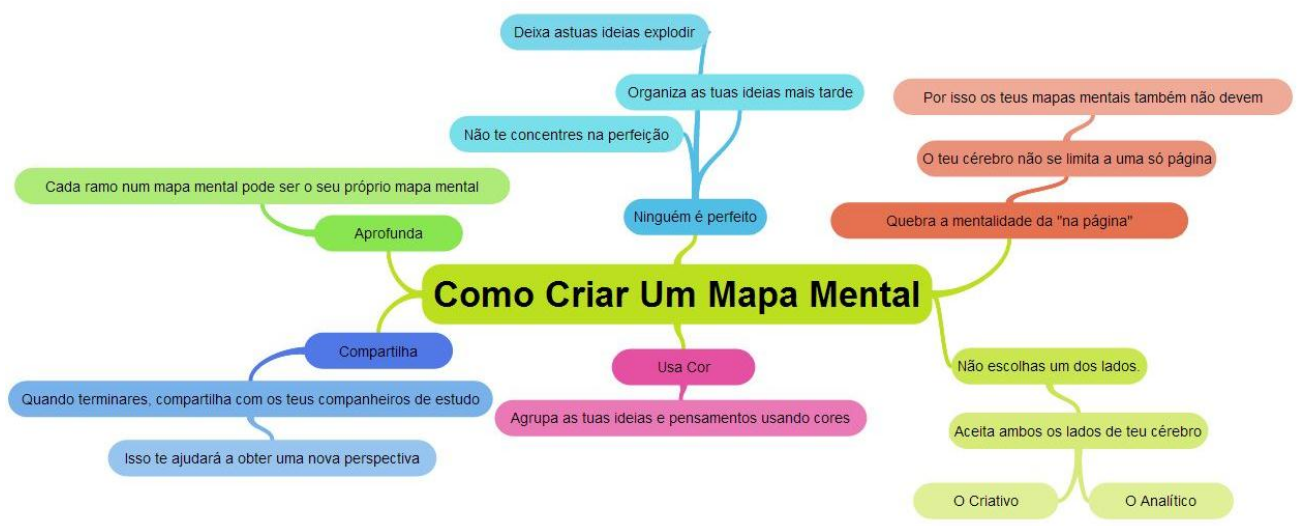

Fig 10 - A estrutura de um mapa mental. (mapasmentais, 2017)

O presente estudo explorou os conceitos através do mapa mental (vide figura 11), e os seus resultados foram obtidos pela análise desse mapa, elaborando as diretrizes de projeto. 


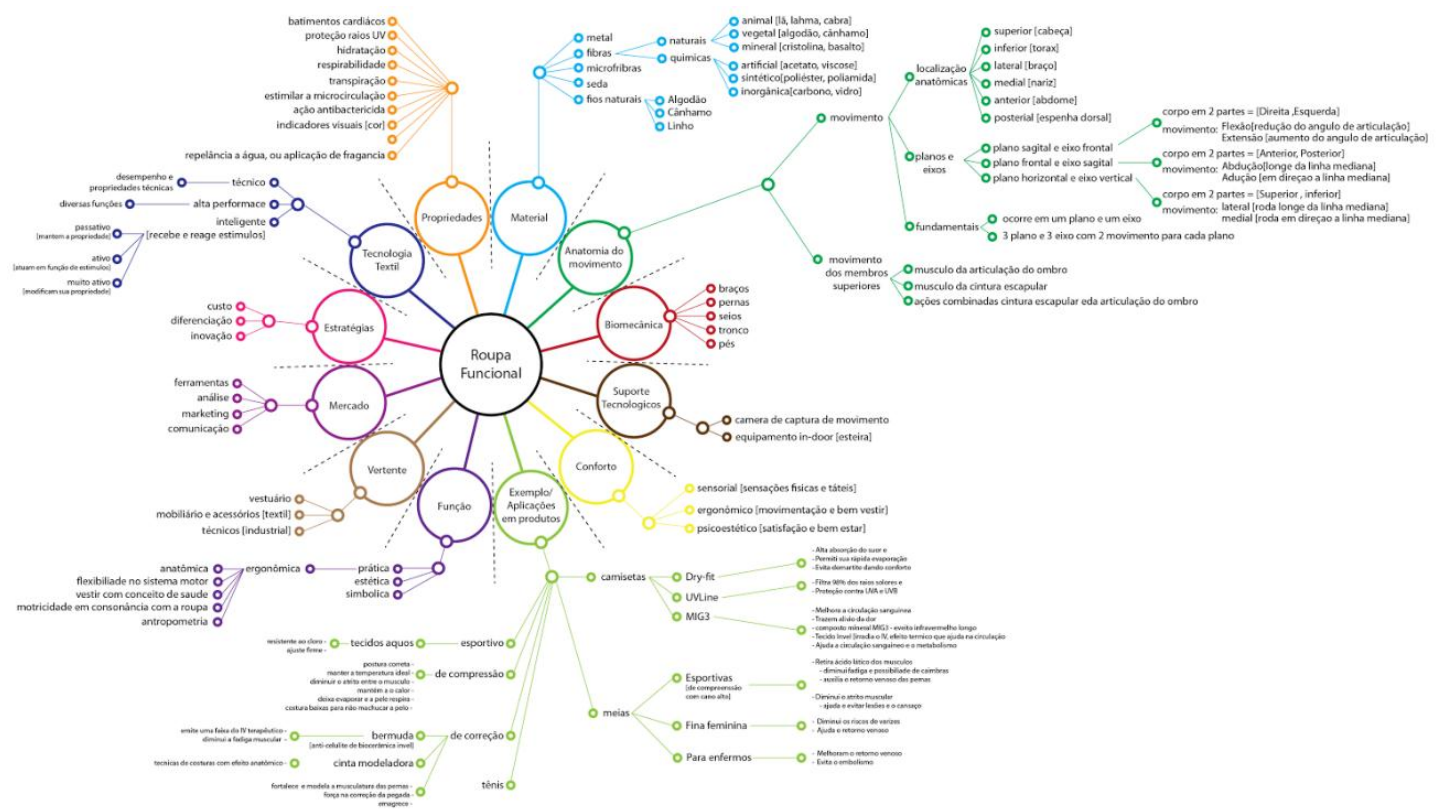

Fig 11 - Mapa mental explorando o tema roupa funcional.

O mapa mental foi construído utilizando pesquisa em revistas, livros, vídeos, etc, realização de entrevistas com as mulheres que praticam esportes, trabalham em fábricas, profissionais da área, etc. A finalidade foi explorar o tema roupa funcional, segundo os aspectos, função, propriedades, tecnologias, biomecânica, material, anatomia do movimento, mercado, produtos, conforto, estratégias.

\subsection{Diretrizes de projeto para roupa funcional}

A construção do mapa mental forneceu base para a análise da área de informação - do material, anatomia do movimento, função, conforto e aplicação em produtos - a partir do qual foram elaboradas as diretrizes de projeto, vide quadro 1 : 
Ourives, Eliete Auxiliadora Assunção; Figueiredo, Attilio Bolivar Ourives de; Figueiredo, Luiz Fernando Gonçalves de; Vieira, Milton Luiz Horn; Moreira, Isabel Cristina Victoria \& Castro,

Quadro 1 - Análise do mapa mental.

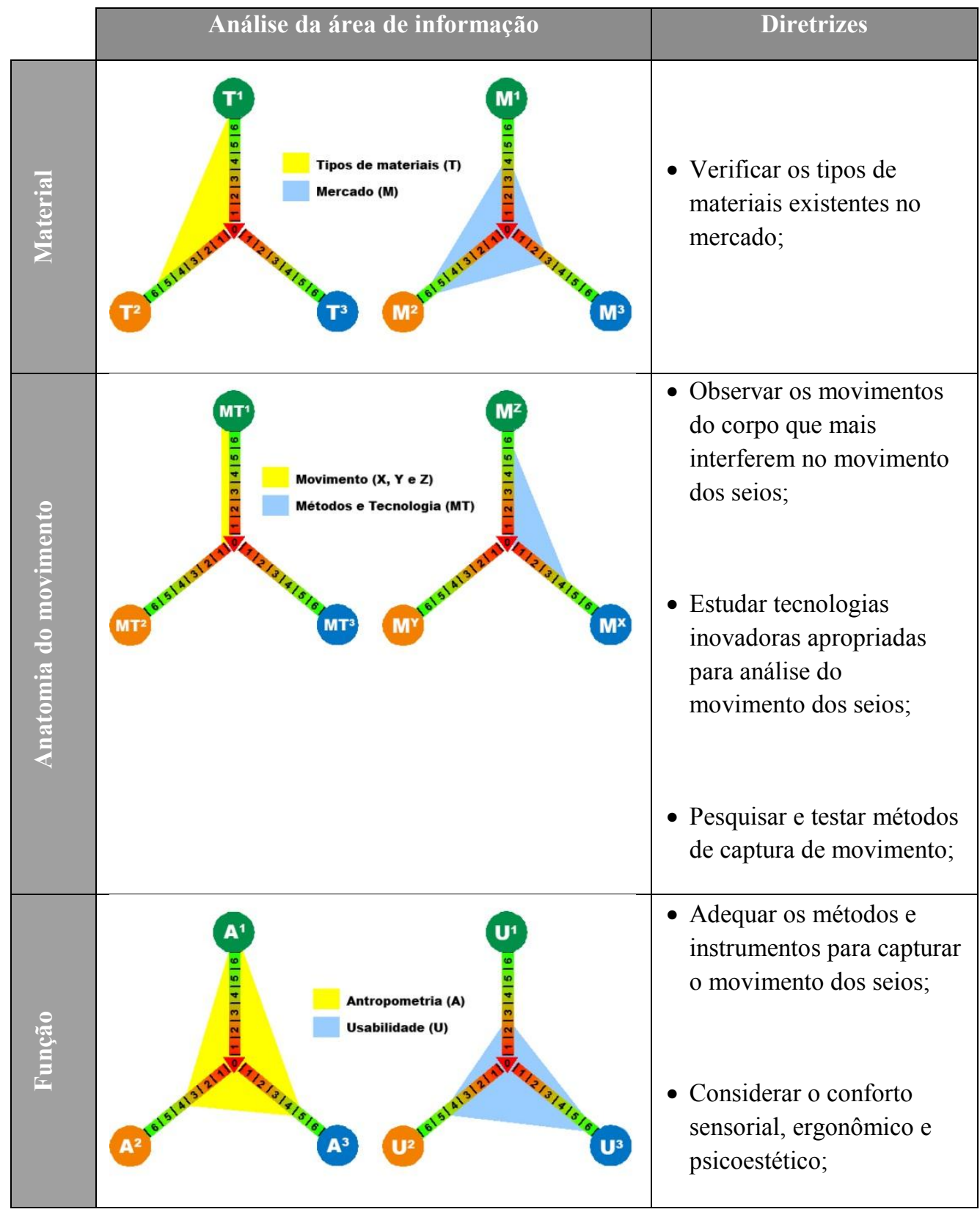




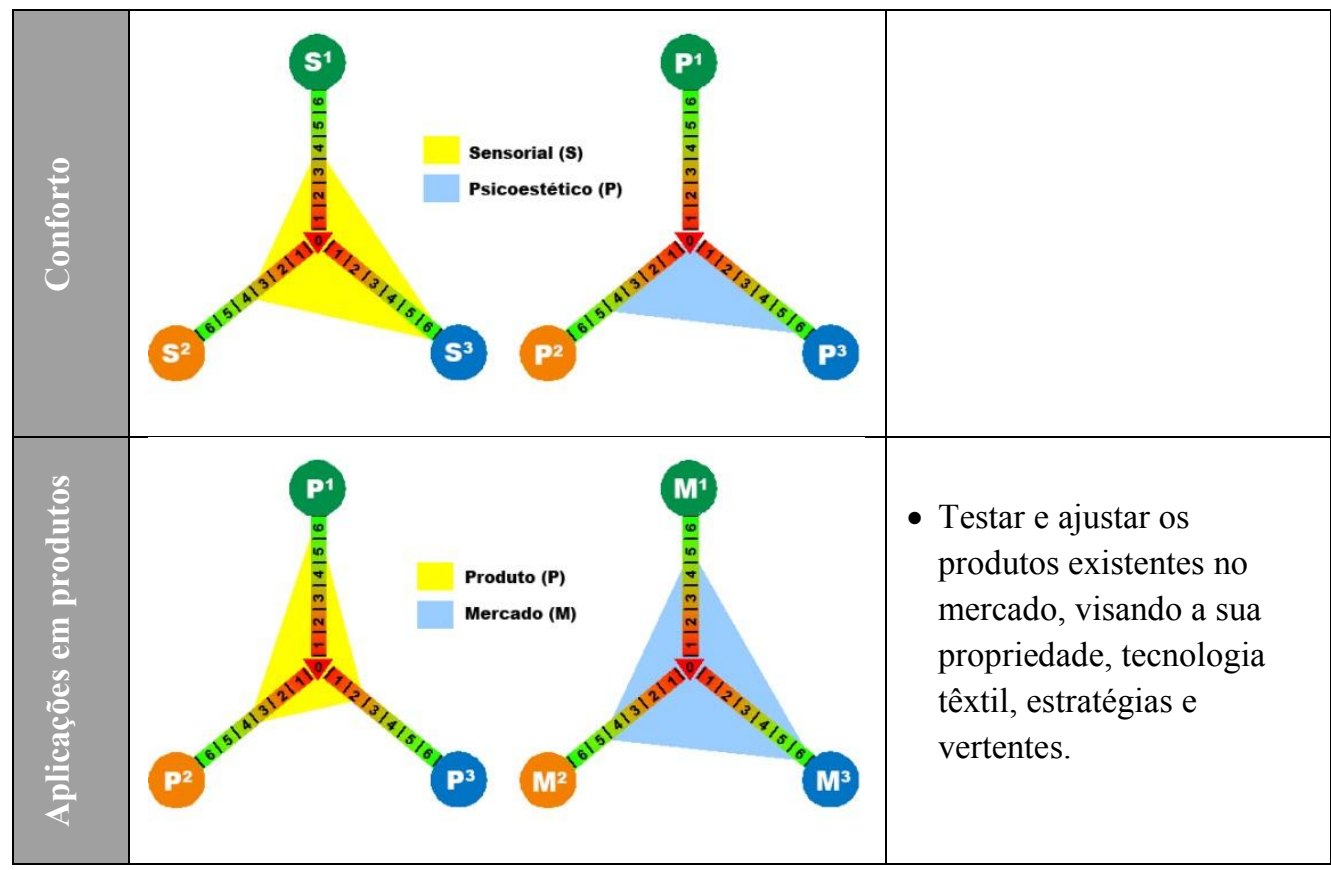

Essas diretrizes serviram como parâmetros para a especificação e elaboração do projeto de roupas funcionais, por exemplo, ao verificar os tipos de materiais disponíveis no mercado observou-se que é possível utilizar as fibras, microfibras e as fibras naturais nas roupas. Além disso, esses tipos de materiais podem ajudar e favorecer o conforto durante o movimento em atividade física.

\section{Conclusão}

A alta tecnologia aplicada ao vestuário estão sempre inovando a cada ano no que se refere às variadas funções para as roupas femininas para usabilidade em trajes esportivos e íntimos. Os avanços em tecidos inteligentes, modelos diferenciados e alta tecnologia prezam sempre pelo bem-estar do usuário.

A criação de roupas funcionais tem que considerar três características fundamentais, a funcionalidade, segurança, bem-estar e conforto das mulheres, tendo em vista as atividades físicas e laborais praticadas por elas. Porém, apesar da grande quantidade de materiais e modelos disponível atualmente no mercado, no que se refere ao vestuário íntimo e esportivo das mulheres, ainda existe insatisfação em relação a funcionalidade e conforto. 
Verifica-se que pesquisar e estruturar as informações sobre o tema, visando requisitos de projeto de roupas funcionais é de grande importância, pois, irá adequar os projetos para atividades no trabalho de fábrica, práticas esportivas, etc.

Nesse aspecto, o uso de mapa mental, mostrou ser uma ferramenta relevante, no sentido de explorar o tema, observando, descobrindo, aprendendo, compreendendo sobre o assunto, no caso deste trabalho, as roupas funcionais, tendo em vista o conforto e bem-estar das mulheres, proporcionando a elaboração diretrizes para cada área de informação que foi considera. Essas diretrizes geradas direcionam ao desenvolvimento de conceitos, ideias, com propostas de projetos, protótipos.

Com base nos estudo desenvolvido neste trabalho sugere-se a continuidade, através: da realização de pesquisa dos produtos e materiais utilizados para confecção de roupas que considere o movimento dos seios femininos na realização de práticas laborais e esportivas; da utilização de métodos e equipamentos específicos para captura de movimentos de seios, visando a produção de roupas funcionais; da comparação de roupas intimas e esportivas existentes no mercado em termos de movimento dos seios durante as práticas laborais e esportivas; da produção de protótipos que inibam o movimento dos seios durante as práticas laborais e esportivas, tendo em vista, o conforto e bem estar das mulheres.

\section{Referencias}

Associação Brasileira de Ergonomia ( 2017, 5 de maio). Recuperado de: www.abergo.org.br/

Aros, K C. Elicitação do processo projetual do Núcleo de Abordagem Sistêmica do Design da Universidade Federal de Santa Catarina. Orientador: Luiz Fernando Gonçalves de Figueiredo - Florianópolis, SC, 2016.

Bertalanffy, L V. (2008) Teoria geral dos sistemas: fundamentos, desenvolvimento e aplicações. Petrópolis : Vozes

Best, K. (2012) Fundamentos de gestão do design. Porto Alegre: Bookman.

Bomfim, G. A. (1995) Metodologia para Desenvolvimento de Projeto. João Pessoa: Universidade federal da Paraíba.

Bonsiepe, G. (2011) Design, Cultura e Sociedade. 1 ed. São Paulo: Blucher.

Buzzan, T. (2005) Mapas Mentais e sua elaboração: um sistema definitivo de pensamento que transforma a sua vida. São Paulo: Cultrix.

Buzzan,T. (2009) Mapas Mentais no trabalho: como ser o melhor na profissão e ainda ter tempo para lazer. São Paulo: Cultrix. 
Dias E. C. (2006) Condições de vida, trabalho, saúde e doença dos trabalhadores rurais no Brasil. (Ed): Saúde do trabalhador rural-RENAST( 1-27) Brasília: Ministério da Saúde.

Ewing, E. (1989) Everyday dress: 1650-1900. David \& Charles.

Gil, A. C. (2010) Como elaborar projetos de pesquisa. São Paulo: Atlas

Gomes, J. (2003) Ergonomia do objeto: sistema técnico de leitura ergonômica. São Paulo: Escrituras Editora.

Guimarães, L. B. M. (2000) Ergonomia de Processo. Porto Alegre:PPGE/UFRGS.

Iida, I. (2005) Ergonomia: projeto e produção. São Paulo: Edgard Blucher.

Krucken, L. (2009) Design e território: valorização de identidades e produtos locais. São Paulo: Studio Nobel.

Labat, K.L.( 2006) Human Factors as Applied in apparel design. (Ed). International Encyclopedia of Ergonomics and Human Factors.( pp 1655-1657) Boca Raton Florida : CRC Press.

Montemezzo, M. C. F. S. (2003) Diretrizes metodológicas para o projeto de produtos de moda no âmbito acadêmico. ( Dissertação inédita de Mestrado) - Universidade Estadual Paulista, Bauru.

Mcghee, D.E.; Steele, J.R.; Munro; J. (2008). Sports bra fitness. Wollongong (NSW): Breast Research Australia.

Mcghee, D.E.; Steele, J.R.; Munro, B.J., (2010). Education improves bra knowledge and fit, and level of breast support in adolescent female athletes: a cluster-randomised trial. Journal of Physiotherapy, 56, 19-24.

Pandarum, R.; Yu, W.; HUNTER, L. (2011). 3-D breast anthropometry of plus-sized women in South Africa. Ergonomics, 54(9), 866-875.

Pechter, E.A. (1998). A new method for determining bra size and predicting postaugmentation breast size. Plastic and Reconstructive Surgery, 102 (4), 1259-1265.

Reilly, T. Ergonomic Considerations for Sports Clothing. ( 2017, 5 de maio) Recuperado em: http:/www.humankinetics.com/excerpts/excerpts/ergonomic-considerations-for-sportsclothing.

Richardson, R. J.(2008) Pesquisa social: métodos e técnicas. 3 ed. São Paulo: Atlas.

Rio, D.; Pires, L. (2001) Ergonomia: fundamentos da prática ergonômica. Editora LTr.

Santos, N.( 1997) Antropotecnologia: A Ergonomia dos sistemas de Produção. Curitiba: Gênesis.

Vasconcellos, M. J. (2013) Pensamento sistêmico: O novo paradigma da ciência. Campinas, SP: Papirus,.

Weerdmeester, J. D. (2001) B. Ergonomia Prática. São Paulo: Edgard Blucher,. 
Ourives, Eliete Auxiliadora Assunção; Figueiredo, Attilio Bolivar Ourives de; Figueiredo, Luiz Fernando Gonçalves de; Vieira, Milton Luiz Horn; Moreira, Isabel Cristina Victoria \& Castro,

Francisco Gómez

White, J.; Scurr, J.; Smith, N. (2009) The effect of breast support on kinetics during overground running performance. Ergonomics, Taylor \& Francis. 52 (4), 492-498.

Labat, K.L.( 2006) Human Factors as Applied in apparel design. (Ed). International Encyclopedia of Ergonomics and Human Factors. ( pp 1655-1657) Boca Raton Florida : CRC Press. 